\section{Relationship between socioeconomic and lifestyle factors and cataracts in Koreans: The Korea National Health and Nutrition Examination Survey 2008-2011}

\begin{abstract}
Purpose Cataracts are the leading cause of visual impairment and blindness, and therefore early identification and modification of the risk factors for cataracts are meaningful. This study aimed to investigate the relationship between socioeconomic status (SES) and lifestyle factors, and age-related cataracts in South Korea.

Methods This cross-sectional study was based on data collected in the 2008-2011 Korea National Health and Nutrition Examination Survey. A total of 15866 subjects, aged $\geq 40$ years, were included. SES was defined using household income and education level. Sociodemographic, lifestyle, and other associated factors were assessed by health interviews and examinations. Cataracts were diagnosed via slit-lamp examination using the Lens Opacities Classification System III.

Results The prevalence of any cataract was $38.9 \%$ in men and $42.3 \%$ in women $(P<0.001)$. In women, the risk of cataracts increased with decreases in household income ( $P$-value for trend $=0.016$ and 0.041 in any, and cortical cataract, respectively) and education level ( $P$-value for trend $=0.009,0.027$, and 0.016 in any, nuclear, and cortical cataract, respectively) after adjusting for confounding factors. Current smoking was correlated with nuclear cataracts in men (OR 1.21; 95\% CI: $1.00,1.46$ in age-adjusted analysis) and cataract surgery in women (OR 2.25; 95\% CI: 1.00, 5.04 in multivariate-adjusted analysis). Conclusions Socioeconomic disparities in cataract prevalence were observed in women; current smoking increased the risk of nuclear cataracts in men and surgery in women.
\end{abstract}

GE Nam¹, K Han², SG Ha ${ }^{3}$, B-D Han ${ }^{1}, \mathrm{DH}$ Kim¹, $\mathrm{Y}-\mathrm{H} \mathrm{Kim}{ }^{1}, \mathrm{KH} \mathrm{Cho}^{1}, \mathrm{YG} \mathrm{Park}^{2}$ and B-J Ko ${ }^{4}$

Public health interventions focusing on gender differences are warranted to prevent and treat cataracts.

Eye (2015) 29, 913-920; doi:10.1038/eye.2015.66; published online 15 May 2015

\section{Introduction}

Cataracts are the leading causes of visual impairment and blindness worldwide, accounting for about half of 37 million blind people. ${ }^{1,2}$ Although the prevalence of blindness due to cataracts was found to vary by country, $>90 \%$ of the total disability adjusted-life years have been reported owing to cataracts, especially in developing countries. ${ }^{3}$ Visual impairment causes a wide spectrum of difficulties in patients' daily living activities, social interactions, and cognitive performance. Furthermore, visual impairment is found to be positively correlated with a risk of falls, and is an independent risk factor of mortality. All of these difficulties contribute to an extensive economic burden to the society. ${ }^{4-6}$

Therefore, early identification and modification of the risk factors for cataracts are meaningful from a public health perspective. A growing body of research has been conducted to investigate risk factors associated with the development and progression of age-related cataracts. Several lifestyle and sociodemographic characteristics, as well as medical conditions such as diabetes, have been reported to affect the risk of cataracts. However, these studies have been performed mainly in Western countries including the United States, European nations, and Australia. ${ }^{7-9}$ Although there has been recent

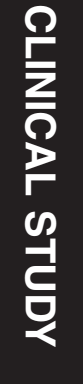

${ }^{1}$ Department of Family Medicine, College of Medicine, Korea University, Seoul, South Korea

${ }^{2}$ Department of Biostatistics, College of Medicine, The Catholic University of Korea, Seoul, South Korea

${ }^{3}$ Department of Ophthalmology, College of Medicine, Korea University, Seoul, South Korea

${ }^{4}$ Total Healthcare Center, Kangbuk Samsung Hospital, Sungkyunkwan University, School of Medicine, Seoul, South Korea

Correspondence: B-J Ko, Total Healthcare Center, Kangbuk Samsung Hospital, Sungkyunkwan University, School of Medicine, 108 Pyung dong, Jongro-Gu, Seoul 110-746, South Korea

Tel: +82 215998113 ; Fax: +82 314125364 . E-mail: basslove1@ hanmail.net or YG Park, Department of Biostatistics, College of Medicine, The Catholic University of Korea, 222 Banpo-daero, Seocho-Gu, Seoul 137-701, South Korea

Tel: +82 22258 7226; Fax: +82314125365. E-mail:ygpark@ catholic.ac.kr

Received: 29 October 2014 Accepted in revised form: 22 February 2015 Published online: 15 May 2015 
data from China and Southeast Asia, including India and Singapore, ${ }^{10-12}$ the data of population-based trends from other Asian countries, including the East Asian countries, have not been fully reported.

Thus, we aimed to investigate the relationship between socioeconomic and lifestyle factors, and cataracts among the South Korean population, based on nationally representative data.

\section{Materials and methods}

\section{Survey overview and study participants}

We analyzed the data from the 2008-2011 Korea National Health and Nutrition Examination Survey (KNHANES) conducted by the Korea Centers for Disease Control and Prevention (KCDC). The KNHANES is a nationwide cross-sectional survey, designed to accurately assess national health and nutrition levels, and consists of a health interview, a health examination, and nutritional assessment. A stratified, multistage, cluster sampling design was used for the selection of subjects for this survey, to represent all the non-institutionalized, civilian population in Korea.

Among the 16014 participants aged $\geq 40$ years, 148 were excluded owing to missing values. Data from 15866 subjects (6833 men and 9033 women) were analyzed. All the study participants provided written informed consent, and the Institutional Review Board of the KCDC approved the study protocol.

\section{Sociodemographic and lifestyle variables}

All participants were asked about their lifestyle and sociodemographic characteristics via interviews by trained staff, or self-report questionnaires. Participants were divided into three groups based on average alcohol intake: non-drinker, light to moderate drinker (1-30 g per day), and heavy drinker ( $>30 \mathrm{~g}$ per day). Participants were categorized as non-smoker, ex-smoker, and current smoker according to their answers on the self-report questionnaire. Participants who exercised moderately more than five times per week for over 30 min per session, or who engaged in vigorous exercise more than three times per week for over $20 \mathrm{~min}$ per session, were considered regular physical exercisers. Residential areas were categorized into urban and rural areas. Participants were also categorized by occupational status (employed in agriculture, forestry, and fishery, or other). In addition, questionnaires were used to assess whether subjects' average exposure to sunlight or ultraviolet light was $<$ or $>5 \mathrm{~h}$ per day.

\section{Socioeconomic variables}

Socioeconomic status (SES) was assessed using household income and education level. Subjects were categorized into quartiles of monthly household income levels: lowest, medium-low, medium-high, and highest.

Education level was classified into four groups: less than elementary school, middle school, high school, and more than college level.

\section{Various associated factors}

Height and body weight were measured, and then body mass index (BMI) was calculated using the formula: body weight $(\mathrm{kg}) /$ height $^{2}\left(\mathrm{~m}^{2}\right)$. Waist circumference (WC) was measured at the midpoint between the lower border of the rib cage and the iliac crest. Systolic and diastolic blood pressures were measured using a standard sphygmomanometer. Blood samples were taken after at least $8 \mathrm{~h}$ of fasting, transported to the Central Testing Institute in Seoul, South Korea. Fasting plasma glucose (FPG) level was measured with a Hitachi Automatic Analyzer 7600 (Hitachi, Tokyo, Japan) by enzymatic methods. The presence of hypertension was defined by blood pressure $\geq 140 / 90 \mathrm{~mm} \mathrm{Hg}$ or by the current use of antihypertensive medication. Diabetes was defined by FPG levels $\geq 126 \mathrm{mg} / \mathrm{dl}$, treatment with insulin or oral agents, or diagnosis by a physician.

\section{Assessment of cataracts}

Subjects underwent a comprehensive eye slit-lamp examination (Haag-Streit model BQ-900; Haag-Streit, Koeniz, Switzerland) by ophthalmologists (second- or third-year resident physicians). Lens Opacities Classification System III (LOCS III) photographic images were used as the reference for grading. ${ }^{13}$ Grading was based on objective measures of color, density, and area, and each lens was assigned an integer grade with values ranging from 0 to $6(0-7$ in the case of nuclear opalescence), and the grading nomenclature of nuclear color, nuclear opalescence, cortical cataract, and posterior subcapsular cataract (PSC) was adopted. Nuclear cataracts were defined by an LOCS III score of $\geq 2$ for nuclear opalescence or $\geq 2$ for nuclear color. Cortical cataracts and PSC were defined as an LOCS III score of $\geq 2$ and of $\geq 1$, respectively. Any cataract was defined as the presence of a nuclear (nuclear opalescence and nuclear color), cortical cataract, or PSC, as well as pseudophakia or aphakia (prior cataract surgery) in at least one eye. Survey participants were classified as having a specific cataract subtype if it was found in either eye; if the subjects showed different cataract subtypes in each eye, 
they were categorized as cases in the analyses of each subtype.

\section{Statistical analyses}

Statistical analyses were performed using SAS version 9.2 for windows (SAS Institute, Cary, NC, USA). A Student's $t$-test or $\chi^{2}$ test was carried out to evaluate the differences in baseline characteristics. The $\chi^{2}$ test was applied to examine the distribution of any cataract, specific cataracts, or surgery according to socioeconomic and lifestyle factors for men and women separately. Interactions of education level and household income for gender were tested before analyses using a logistic regression model. The association of socioeconomic or lifestyle factors with risk of cataracts was assessed by age- and multivariateadjusted logistic regression analyses. The odds ratios (ORs) and 95\% confidence intervals (CIs) were estimated after adjusting for the variables including age, household income, education level, alcohol consumption, smoking status, physical activity, BMI, diabetes, hypertension, history of cardiovascular disease or stroke, hormone replacement therapy (women), occupation, residential area, and sun exposure duration. All estimates were weighted to allow for oversampling, non-response, and the Korean population in 2008-2011, taking the complex design of the survey into consideration. A two-sided $P<0.05$ was regarded as statistically significant.

\section{Results}

Table 1 presents the baseline for subjects according to gender and cataract status. The mean age, the proportion of chronic diseases, residence in rural area, outdoor occupations, and sun exposure duration over $5 \mathrm{~h}$ were higher in subjects with cataracts than those without cataracts, in both genders.

The distribution of cataract prevalence according to socioeconomic and lifestyle subgroups is shown in Table 2. The prevalences of any, nuclear, cortical cataract, PSC, and cataract surgery were 38.9\%, 25.6\%, 12.4\%, $0.6 \%$, and $9.3 \%$ in men and $42.3 \%, 27.5 \%, 12.2 \%, 0.8 \%$, and $12.9 \%$ in women, respectively. The proportions of all types of cataracts decreased with higher SES in both genders. The prevalences of all types of cataracts decreased with increases in the level of alcohol consumption in both genders except for PSC in men. Compared with current smokers, the prevalences of all types of cataracts was higher in non- or ex-smokers in men; in contrast, the proportions of surgery was higher in current smokers in women $(P=0.012)$. As for physical activity, the prevalence of any, nuclear cataracts, and surgery were significantly higher in those who did not exercise regularly, compared with those who exercised regularly, in both genders.

There were differences of education level $(P<0.001)$ and household income $(P<0.001)$ for genders; therefore, the data were analyzed separately according to gender. Table 3 shows the risk factors of cataracts according to socioeconomic and lifestyle factors in men and women after adjusting for confounding variables. In men, the risk of nuclear cataracts significantly increased in current smokers, compared with non-smokers or ex-smokers, only in age-adjusted analysis (OR 1.21; 95\% CI: 1.00, 1.46). In women, the risk of any, nuclear, and cortical cataracts increased as household income level decreased, in ageadjusted analyses. These trends persisted with any and cortical cataracts after adjusting for all confounders $(P$ value for trend $=0.016$ and 0.041 in any and cortical cataract, respectively). The ORs of any, nuclear, cortical cataracts, and surgery increased steadily as education level decreased in age-adjusted analyses. In multivariateadjusted analyses, these negative associations remained significant only with any, nuclear, and cortical cataracts ( $P$-value for trend $=0.009,0.027$, and 0.016 , respectively). The OR of surgery increased in current smokers in women in multivariate-adjusted analyses (OR 2.25; 95\% CI: $1.00,5.04)$. The overall risk of cataracts in women did not differ significantly according to lifestyle factors such as alcohol consumption, smoking status, and physical activity.

\section{Discussion}

Low SES was associated with high prevalence of any, nuclear, and cortical cataracts in women, whereas the association between SES and cataracts was not observed in men. Moreover, the risk for cataracts showed an increasing trend with decreasing SES levels in women. Current smoking increased the risk of nuclear cataracts in men and surgery in women.

Worldwide, increasing evidence has reported that low SES is associated with both the prevalence and progression of cataracts. In the United States and Europe, higher education levels were associated with a decreased risk of cataracts in a prospective cohort study. 8,14 Income or education levels were inversely related to the 10-year cumulative incidence of nuclear cataracts in the Beaver Dam Eye Study. ${ }^{7}$ Similar trends were also observed in studies performed in Asian countries such as Singapore, ${ }^{11,15}$ India, ${ }^{12}$ China, ${ }^{10}$ and Bangladesh. ${ }^{16}$

The reason for the association between low SES and the prevalence of cataracts is yet to be elucidated, although several possible explanations have been suggested. People with low SES may not be able to afford to have cataract surgery, and this could lead to higher prevalence of cataracts. However, in accordance with a previous 
Table 1 General characteristics of study subjects in the 2008-2011 KNHANES ${ }^{a}$

\begin{tabular}{|c|c|c|c|c|c|c|}
\hline \multirow{2}{*}{ Characteristic } & \multicolumn{3}{|c|}{$\operatorname{Men}(\mathrm{n}=6833)$} & \multicolumn{3}{|c|}{ Women $(\mathrm{n}=9033)$} \\
\hline & Any cataract & No cataract & $\mathrm{P}-$-value $e^{\mathrm{b}}$ & Any cataract & No cataract & P-value ${ }^{\mathrm{b}}$ \\
\hline$N$ & 3351 & 3482 & & 4431 & 4602 & \\
\hline Age (years) & $62.9(0.3)$ & $49.5(0.2)$ & $<0.001$ & $66.1(0.3)$ & $49.7(0.2)$ & $<0.001$ \\
\hline BMI $\left(\mathrm{kg} / \mathrm{m}^{2}\right)$ & $23.6(0.1)$ & $24.3(0.1)$ & $<0.001$ & $24.1(0.1)$ & $23.8(0.1)$ & 0.006 \\
\hline $\mathrm{WC}(\mathrm{cm})$ & $85.1(0.2)$ & $85.2(0.2)$ & 0.924 & $82.5(0.2)$ & $79.3(0.2)$ & $<0.001$ \\
\hline Hypertension (\%) & $51.2(1.2)$ & $37.8(1.0)$ & $<0.001$ & $55.7(1.1)$ & $23.9(0.8)$ & $<0.001$ \\
\hline Diabetes (\%) & $20.5(0.9)$ & $11.2(0.7)$ & $<0.001$ & $18.5(0.9)$ & $6.3(0.5)$ & $<0.001$ \\
\hline Hx of CVD or stroke (\%) & $7.9(0.6)$ & $2.7(0.3)$ & $<0.001$ & $5.9(0.4)$ & $1.8(0.2)$ & $<0.001$ \\
\hline Household income level (\%) & & & $<0.001$ & & & $<0.001$ \\
\hline Lowest & $30.5(1.1)$ & $11.0(0.6)$ & & $41.8(1.1)$ & $13.0(0.7)$ & \\
\hline Medium-low & $26.5(1.0)$ & $24.7(1.0)$ & & $24.8(0.8)$ & $26.7(0.9)$ & \\
\hline Medium-high & $21.7(1.0)$ & $30.5(0.9)$ & & $18.2(0.8)$ & $28.5(0.8)$ & \\
\hline Highest & $21.4(1.1)$ & $33.8(1.1)$ & & $15.2(0.8)$ & $31.7(1.1)$ & \\
\hline Education level (\%) & & & $<0.001$ & & & $<0.001$ \\
\hline$\leq$ Elementary school & $35.7(1.2)$ & $12.8(0.7)$ & & $70.9(1.1)$ & $22.6(0.9)$ & \\
\hline Middle school & $18.5(0.9)$ & $15.3(0.7)$ & & $11.7(0.7)$ & $18.2(0.7)$ & \\
\hline High school & $27.6(1.0)$ & $37.6(1.1)$ & & $14.0(0.8)$ & $39.8(1.0)$ & \\
\hline$\geq$ College & $18.2(1.1)$ & $34.3(1.2)$ & & $3.5(0.4)$ & $19.5(0.9)$ & \\
\hline Alcohol consumption (\%) & & & $<0.001$ & & & $<0.001$ \\
\hline Non-drinker & $26.1(1.0)$ & $15.1(0.7)$ & & $56.6(1.0)$ & $34.1(0.9)$ & \\
\hline Light to moderate drinker & $57.3(1.1)$ & $64.2(1.0)$ & & $42.9(1.0)$ & $64.4(0.9)$ & \\
\hline Heavy drinker & $16.7(0.9)$ & $20.7(0.8)$ & & $0.5(0.1)$ & $1.5(0.2)$ & \\
\hline Smoking status (\%) & & & $<0.001$ & & & 0.496 \\
\hline Non- or ex-smoker & $65.3(1.1)$ & $56.1(1.1)$ & & $95.3(0.4)$ & $95.7(0.4)$ & \\
\hline Current smoker & $34.7(1.1)$ & $43.9(1.1)$ & & $4.7(0.4)$ & $4.3(0.4)$ & \\
\hline Regular exerciser (\%) & $23.3(1.1)$ & $27.4(1.0)$ & 0.003 & $18.8(0.9)$ & $23.8(0.8)$ & $<0.001$ \\
\hline Residential area (rural) & $28.7(2.4)$ & $21.9(1.8)$ & 0.001 & $31.3(2.3)$ & $20.0(1.7)$ & $<0.001$ \\
\hline Occupation (agriculture, forestry and fishery) & $16.6(1.5)$ & $9.3(1.0)$ & $<0.001$ & $11.1(1.1)$ & $5.8(0.6)$ & $<0.001$ \\
\hline Sun exposure duration $\geq 5 \mathrm{~h}$ & $34.1(1.5)$ & $27.5(1.1)$ & $<0.001$ & $22.7(1.4)$ & $12.2(0.7)$ & $<0.001$ \\
\hline Ever use of HRT & - & - & - & $9.9(0.5)$ & $10.6(0.5)$ & 0.348 \\
\hline
\end{tabular}

Abbreviations: BMI, body mass index; CVD, cardiovascular disease; HRT, hormone replacement therapy; WC, waist circumference. ${ }^{\text {a }}$ Data from $2008-2011$ Korea National Health and Nutrition Examination Survey for adults at least 40 years old are presented as mean (SEM) or percentage (SE). ${ }^{\mathrm{b}} \mathrm{Obtained}$ by Student's $t$-test or $\chi^{2}$ test.

report, ${ }^{10}$ no association between SES and surgery was observed in the present study (Table 3), which suggests that this explanation is unconvincing, and is not consistent with the results of the study.

Low SES may influence a diversity of lifestyle factors, including smoking, drinking, physical activity, and diet quality, as well as environmental exposure to sunlight and indoor cooking smoke, in comparison with higher SES. Differences in the distribution of outdoor occupations and residential areas may also be affected by SES levels. We, therefore, have considered smoking, drinking, physical activity, sunlight exposure, outdoor occupation, and residential area as variables confounding the relationship between SES and cataracts in a multivariate model; however, the association remains significant in women. The intake of antioxidant nutrients, such as $\beta$-carotene, lutein, vitamin A, C, and E, has been revealed as a protective factor against the development of age-related cataracts in several reports. ${ }^{17,18}$ We further analyzed the nutritional intake of study subjects according to the SES, and found that the intakes of carotene, vitamin $\mathrm{A}$, and $\mathrm{C}$ were higher with increasing SES level in women (see Supplementary Tables 1 and 2). Diet of women with low SES in this survey contained less antioxidant nutrients than those with high SES, and may lead to a higher prevalence of all types of cataracts in this group.

The prevalence of cataracts was higher in women $(42.3 \%)$ than in men $(38.9 \%)$, which is in line with previous studies, ${ }^{11,12,19}$ and could be explained by the difference in environmental exposure, and the results of hormonal changes associated with menopause. ${ }^{20}$ 
Table 2 Distribution of any cataract and specific cataract subtypes by socioeconomic and lifestyle factors in the 2008-2011 KNHANES

\begin{tabular}{|c|c|c|c|c|c|c|c|c|c|c|}
\hline \multirow[t]{2}{*}{ Socioeconomic or lifestyle factor } & \multicolumn{5}{|c|}{ Men } & \multicolumn{5}{|c|}{ Women } \\
\hline & Any & Nuclear & Cortical & PSC & Surgery & Any & Nuclear & Cortical & PSC & Surgery \\
\hline$N$ & 3351 & 1754 & 736 & 37 & 591 & 4431 & 2205 & 877 & 51 & 912 \\
\hline \multicolumn{11}{|l|}{ Household income level (\%) } \\
\hline Lowest & $63.7(1.7)$ & $45.2(2.2)$ & $27.2(2.2)$ & $1.8(0.6)$ & $28.2(1.9)$ & $69.9(1.4)$ & $52.3(1.9)$ & $29.1(2.1)$ & $2.2(0.6)$ & $36.7(1.8)$ \\
\hline Medium-low & $40.4(1.7)$ & $28.3(1.8)$ & $12.2(1.3)$ & $1.0(0.4)$ & $8.4(0.8)$ & $40.1(1.4)$ & $26.7(1.5)$ & $13.1(1.1)$ & $0.8(0.2)$ & $9.5(0.8)$ \\
\hline Medium-high & $31.1(1.6)$ & $20.4(1.5)$ & $9.5(1.3)$ & $0.3(0.1)$ & $6.0(0.8)$ & $31.6(1.5)$ & $20.8(1.5)$ & $6.7(0.8)$ & $0.4(0.2)$ & $7.8(0.8)$ \\
\hline Highest & $28.6(1.5)$ & $18.2(1.4)$ & $9.5(1.1)$ & $0.4(0.2)$ & $4.7(0.7)$ & $25.8(1.3)$ & $16.1(1.2)$ & $6.2(0.8)$ & $0.3(0.2)$ & $5.5(0.6)$ \\
\hline$P$-value for trend & $<0.001$ & $<0.001$ & $<0.001$ & 0.004 & $<0.001$ & $<0.001$ & $<0.001$ & $<0.001$ & $<0.001$ & $<0.001$ \\
\hline \multicolumn{11}{|l|}{ Education level (\%) } \\
\hline$\leq$ Elementary school & $63.8(1.8)$ & $47.7(2.3)$ & $27.0(2.3)$ & $2.0(0.7)$ & $25.6(2.0)$ & $69.4(1.2)$ & $52.2(1.7)$ & $29.0(1.6)$ & $2.5(0.6)$ & $34.9(1.4)$ \\
\hline Middle school & $43.4(2.0)$ & $28.0(2.2)$ & $16.8(1.9)$ & $0.8(0.4)$ & $9.5(1.2)$ & $31.7(1.8)$ & $21.6(1.8)$ & $9.9(1.3)$ & $0.1(0.1)$ & $5.2(0.8)$ \\
\hline High school & $31.7(1.5)$ & $22.1(1.5)$ & $8.5(0.9)$ & $0.5(0.2)$ & $6.5(0.6)$ & $20.2(1.2)$ & $13.6(1.1)$ & $4.8(0.6)$ & $0.4(0.2)$ & $2.6(0.4)$ \\
\hline$\geq$ College & $25.1(1.4)$ & $14.9(1.3)$ & $7.7(1.0)$ & $0.3(0.2)$ & $4.6(0.7)$ & $11.4(1.1)$ & $7.5(1.0)$ & $2.3(0.5)$ & $0.1(0.1)$ & $1.4(0.4)$ \\
\hline$P$-value for trend & $<0.001$ & $<0.001$ & $<0.001$ & 0.001 & $<0.001$ & $<0.001$ & $<0.001$ & $<0.001$ & $<0.001$ & $<0.001$ \\
\hline \multicolumn{11}{|l|}{ Alcohol consumption (\%) } \\
\hline Non-drinker & $52.4(1.9)$ & $36.8(2.1)$ & $17.0(1.8)$ & $0.7(0.3)$ & $17.9(1.5)$ & $54.9(1.1)$ & $36.6(1.3)$ & $18.2(1.2)$ & $1.2(0.3)$ & $22.9(1.1)$ \\
\hline Light to moderate drinker & $36.2(1.2)$ & $23.7(1.2)$ & $11.5(0.9)$ & $0.7(0.2)$ & $8.2(0.6)$ & $32.8(1.1)$ & $21.8(1.2)$ & $8.8(0.7)$ & $0.6(0.2)$ & $6.8(0.5)$ \\
\hline Heavy drinker & $33.9(1.9)$ & $21.8(1.8)$ & $11.8(1.4)$ & $0.4(0.2)$ & $5.9(0.9)$ & $21.2(4.3)$ & $16.6(3.9)$ & $4.7(2.7)$ & - & $1.4(1.0)$ \\
\hline$P$-value for trend & $<0.001$ & $<0.001$ & 0.010 & 0.317 & $<0.001$ & $<0.001$ & $<0.001$ & $<0.001$ & 0.011 & $<0.001$ \\
\hline \multicolumn{11}{|l|}{ Smoking status (\%) } \\
\hline Non- or ex-smoker & $42.3(1.2)$ & $27.9(1.3)$ & $14.1(1.0)$ & $0.9(0.2)$ & $11.8(0.7)$ & $41.8(1.0)$ & $27.2(1.1)$ & $12.2(0.7)$ & $0.8(0.2)$ & $12.5(0.5)$ \\
\hline Current smoker & $33.3(1.4)$ & $22.2(1.4)$ & $10.0(1.0)$ & $0.3(0.1)$ & $5.8(0.6)$ & $44.1(3.4)$ & $28.7(3.4)$ & $8.5(1.9)$ & $0.9(0.8)$ & $16.4(2.7)$ \\
\hline$P$-value for trend & 0.001 & 0.046 & 0.033 & 0.020 & $<0.001$ & 0.157 & 0.310 & 0.077 & 0.836 & 0.012 \\
\hline \multicolumn{11}{|l|}{ Physical activity (\%) } \\
\hline Regular exerciser & $34.8(1.7)$ & $22.6(1.7)$ & $11.9(1.2)$ & $0.8(0.3)$ & $6.6(0.8)$ & $36.3(1.5)$ & $24.2(1.7)$ & $11.1(1.1)$ & $0.4(0.2)$ & $8.2(0.8)$ \\
\hline Non-regular exerciser & $40.0(1.1)$ & $26.5(1.2)$ & $12.5(1.0)$ & $0.6(0.1)$ & $10.3(0.6)$ & $43.4(1.0)$ & $28.1(1.1)$ & $12.3(0.8)$ & $0.9(0.2)$ & $14.0(0.6)$ \\
\hline$P$-value for trend & 0.003 & 0.020 & 0.657 & 0.456 & $<0.001$ & $<0.001$ & 0.013 & 0.305 & 0.147 & $<0.001$ \\
\hline
\end{tabular}

Abbreviation: PSC, posterior subcapsular cataract. ${ }^{\text {a }}$ Data are presented as percentage (SE).

Moreover, we found that the association between low SES and the high proportion of cataracts was observed only in women. Exposure to indoor cooking smoke, which is mainly from cheap solid fuels such as wood, coal, and animal dung, increases the risk for cataracts by inducing oxidative stress on the eye. ${ }^{21}$ Women with lower SES may use, or have used, such material more frequently in cooking than those with higher SES, especially the elderly, who have experienced colonization by Japan (1910-1945), the Korean War (1950-1953), and post-war reconstruction (1954-1964). However, we did not examine the information about current and/or past fuel and stove use.

Several studies from Western countries, 7,9,14,19,2 as well as Asian countries, ${ }^{10-12,15}$ have demonstrated the positive relationship between cigarette smoking and the prevalence and/or progression of cataracts. Moreover, a dose-response relationship has been demonstrated between smoking and the prevalence of cataracts. ${ }^{11,12}$ Smoking could affect the lens and retina by promoting atherosclerosis of ocular capillaries and oxidative stress by reactive oxygen species generation. ${ }^{23}$ We initially found that the crude prevalence of cataracts was higher in non- or ex-smokers than in current smokers in men (Table 2); however, after adjusting for age, current male smokers had an increased risk for nuclear cataracts (Table 3), which suggests a strong effect of age on cataracts. We also found that current smoking increased the risk for surgery in women. These findings support the notion that efforts to reduce the smoking rate should be carried out by public health authorities.

Alcohol consumption was not related to cataracts after adjusting for age in this study. The association between cataracts and alcohol consumption has been investigated in cross-sectional, case-control, and longitudinal studies with inconsistent results. ${ }^{7,21,22}$ A study in Australia has shown that alcohol consumption was positively associated with nuclear cataracts, whereas an inverse relationship was observed between alcohol intake and cortical cataracts. ${ }^{22}$ However, no significant trend was found between alcohol intake and the incidence of all types of cataracts in the other papers. $7,11,24$ 


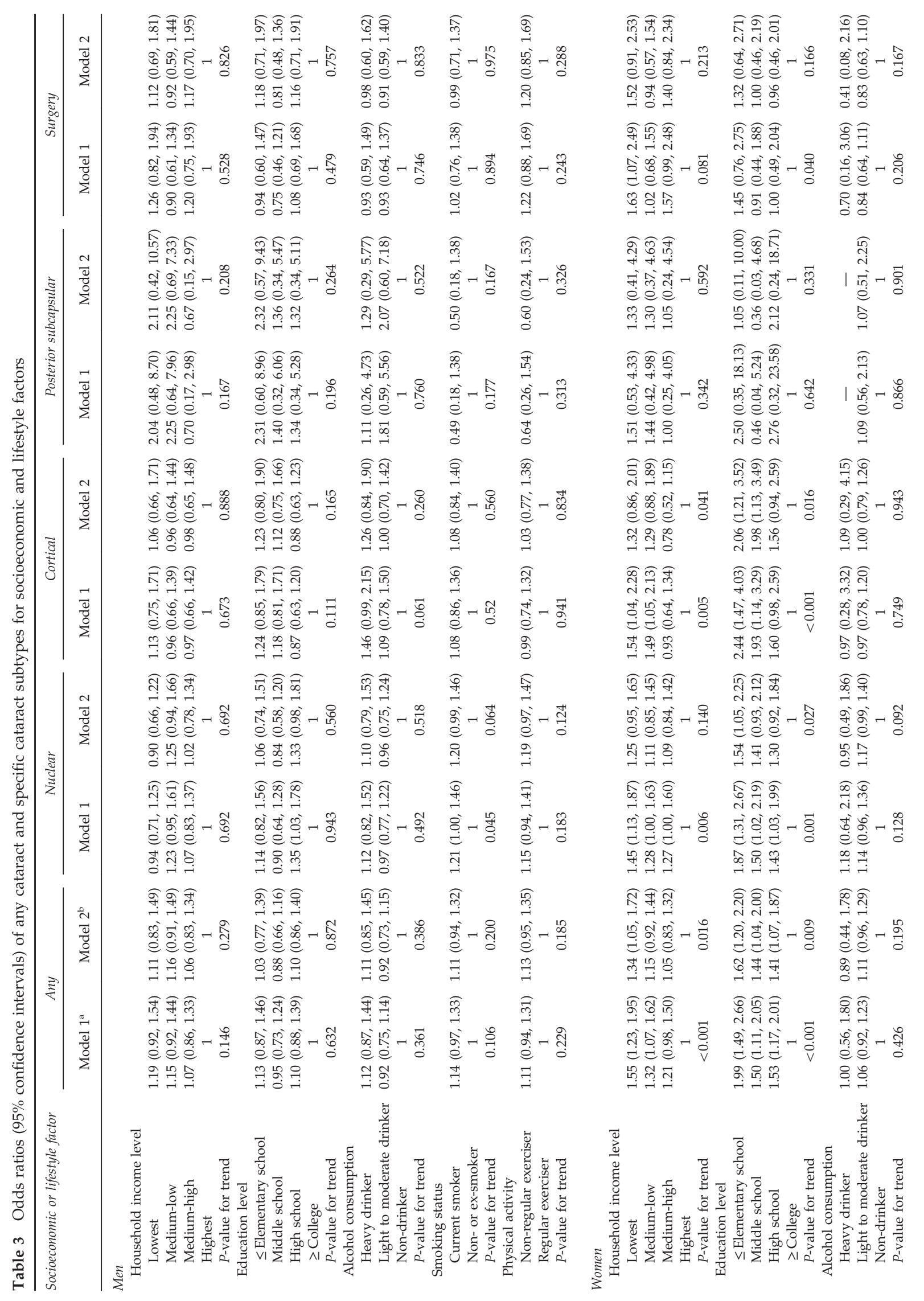


There has been a dearth of investigations regarding the relationship between physical activity and the risk of cataracts. A 7-year follow-up study has revealed that the risk of self-reported cataracts decreased with lower BMI, higher physical activity, and greater cardiorespiratory fitness in men. ${ }^{25}$ However, we could not find a relationship between physical activity and the risk of cataracts, which will need confirmation in future research.

The present study has several limitations. The crosssectional design of our study could not deduce causal relationships between socioeconomic and lifestyle factors and cataracts. We could not consider subjects' medication use, including glucocorticoids, nonsteroidal antiinflammatory drugs, thyroid hormone, and multivitamins, as well as exposure to indoor cooking smoke, and refractory error; all factors might affect the occurrence of cataracts. All of the cataracts diagnosed in this study may not be visually disabling and may not need any surgical intervention. Moreover, the diagnosis of cataracts might differ by the artifact of the photographic image or inter- or intraexaminer reliability of measurements. However, this is the first epidemiologic study focusing on the relationship between socioeconomic and lifestyle factors, and the risk of cataracts in Korea, using nationally representative data.

In conclusion, SES showed a significantly inverse association with the risk of cataracts in Korean women. Moreover, current smoking was correlated with nuclear cataracts in men and surgery in women. Gender-specific public health interventions, considering SES and current smoking status, are needed for the prevention and treatment of cataracts, and to ultimately prevent visual impairment.

\section{Summary}

What was known before

- Low SES has been reported to be associated with both the prevalence and progression of cataracts worldwide.

- The positive relationship between cigarette smoking and the prevalence and/or progression of cataracts has been demonstrated.

- The association between cataracts and alcohol consumption has been reported with inconsistent results and there has been a dearth of investigations regarding the relationship between physical activity and the risk of cataracts.

- The data of population-based trends from the East Asian countries has not been fully reported.

What this study adds

- Socioeconomic disparities in cataract prevalence were observed in Korean women.

- The risk of cataracts increased with decreases in household income and education level in women.

- Current smoking was associated with increased the risk of nuclear cataracts in men and surgery in women. 


\section{Conflict of interest}

The authors declare no conflict of interest.

\section{Acknowledgements}

We thank the participants in the Korea National Health and Nutrition Examination Survey 2008-2011.

\section{References}

1 Pizzarello L, Abiose A, Ffytche T, Duerksen R, Thulasiraj R, Taylor H et al. VISION 2020: The Right to Sight: a global initiative to eliminate avoidable blindness. Arch Ophthalmol 2004; 122: 615-620.

2 Resnikoff S, Pascolini D, Etya'ale D, Kocur I, Pararajasegaram R, Pokharel GP et al. Global data on visual impairment in the year 2002. Bull World Health Organ 2004; 82: 844-851.

3 Rao GN, Khanna R, Payal A. The global burden of cataract. Curr Opin Ophthalmol 2011; 22: 4-9.

4 Rein DB, Zhang P, Wirth KE, Lee PP, Hoerger TJ, McCall N et al. The economic burden of major adult visual disorders in the United States. Arch Ophthalmol 2006; 124: 1754-1760.

5 Knudtson MD, Klein BE, Klein R. Biomarkers of aging and falling: the Beaver Dam eye study. Arch Gerontol Geriatr 2009; 49: $22-26$.

6 Knudtson MD, Klein BE, Klein R. Age-related eye disease, visual impairment, and survival: the Beaver Dam Eye Study. Arch Ophthalmol 2006; 124: 24-29.

7 Klein BE, Klein R, Lee KE, Meuer SM. Socioeconomic and lifestyle factors and the 10-year incidence of age-related cataracts. Am J Ophthalmol 2003; 136: 506-512.

8 Navarro Esteban JJ, Gutierrez Leiva JA, Valero Caracena N, Buendia Bermejo J, Calle Puron ME, Martinez Vizcaino VJ. Prevalence and risk factors of lens opacities in the elderly in Cuenca, Spain. Eur J Ophthalmol 2007; 17: 29-37.

9 Tan JS, Wang JJ, Younan C, Cumming RG, Rochtchina E, Mitchell P. Smoking and the long-term incidence of cataract: the Blue Mountains Eye Study. Ophthalmic Epidemiol 2008; 15: $155-161$.

10 Xu L, Cui T, Zhang S, Sun B, Zheng Y, Hu A et al. Prevalence and risk factors of lens opacities in urban and rural Chinese in Beijing. Ophthalmology 2006; 113: 747-755.

11 Wu R, Wang JJ, Mitchell P, Lamoureux EL, Zheng Y, Rochtchina E et al. Smoking, socioeconomic factors, and age-related cataract: the Singapore Malay Eye study. Arch Ophthalmol 2010; 128: 1029-1035.

12 Krishnaiah S, Vilas K, Shamanna BR, Rao GN, Thomas R, Balasubramanian D. Smoking and its association with cataract: results of the Andhra Pradesh eye disease study from India. Invest Ophthalmol Vis Sci 2005; 46: 58-65.
13 Chylack Jr LT, Wolfe JK, Singer DM, Leske MC, Bullomore MA, Bailey IL et al. The Lens Opacities Classification System III. The Longitudinal Study of Cataract Study Group. Arch Ophthalmol 1993; 111: 831-836.

14 Chang JR, Koo E, Agron E, Hallak J, Clemons T, Azar D et al. Risk factors associated with incident cataracts and cataract surgery in the Age-related Eye Disease Study (AREDS): AREDS report number 32. Ophthalmology 2011; 118: 2113-2119.

15 Foster PJ, Wong TY, Machin D, Johnson GJ, Seah SK. Risk factors for nuclear, cortical and posterior subcapsular cataracts in the Chinese population of Singapore: the Tanjong Pagar Survey. Br J Ophthalmol 2003; 87: 1112-1120.

16 Polack S, Kuper H, Wadud Z, Fletcher A, Foster A. Quality of life and visual impairment from cataract in Satkhira district, Bangladesh. Br J Ophthalmol 2008; 92: 1026-1030.

17 Tan AG, Mitchell P, Flood VM, Burlutsky G, Rochtchina E, Cumming RG et al. Antioxidant nutrient intake and the long-term incidence of age-related cataract: the Blue Mountains Eye Study. Am J Clin Nutr 2008; 87: 1899-1905.

18 Cui YH, Jing CX, Pan HW. Association of blood antioxidants and vitamins with risk of age-related cataract: a metaanalysis of observational studies. Am J Clin Nutr 2013; 98: 778-786.

19 Richter GM, Choudhury F, Torres M, Azen SP, Varma RLos Angeles Latino Eye Study Group. Risk factors for incident cortical, nuclear, posterior subcapsular, and mixed lens opacities: the Los Angeles Latino eye study. Ophthalmology 2012; 119: 2040-2047.

20 Worzala K, Hiller R, Sperduto RD, Mutalik K, Murabito JM, Moskowitz $\mathrm{M}$ et al. Postmenopausal estrogen use, type of menopause, and lens opacities: the Framingham studies. Arch Intern Med 2001; 161: 1448-1454.

21 Pokhrel AK, Smith KR, Khalakdina A, Deuja A, Bates MN. Case-control study of indoor cooking smoke exposure and cataract in Nepal and India. Int J Epidemiol 2005; 34: 702-708.

22 Cumming RG, Mitchell P. Alcohol, smoking, and cataracts: the Blue Mountains Eye Study. Arch Ophthalmol 1997; 115: 1296-1303.

23 Shalini VK, Luthra M, Srinivas L, Rao SH, Basti S, Reddy M et al. Oxidative damage to the eye lens caused by cigarette smoke and fuel smoke condensates. Indian J Biochem Biophys 1994; 31: 261-266.

24 Kanthan GL, Mitchell P, Burlutsky G, Wang JJ. Alcohol consumption and the long-term incidence of cataract and cataract surgery: the Blue Mountains Eye Study. Am J Ophthalmol 2010; 150: 434-440.e1.

25 Williams PT. Prospective epidemiological cohort study of reduced risk for incident cataract with vigorous physical activity and cardiorespiratory fitness during a 7-year follow-up. Invest Ophthalmol Vis Sci 2009; 50: 95-100.

Supplementary Information accompanies this paper on Eye website (http://www.nature.com/eye) 